NASA Technical Memorandum 106450

AIAA-94-0186

\title{
Effect of 'Delta Tabs' on Mixing and Axis Switching in Jets From Asymmetric Nozzles
}

K.B.M.Q. Zaman

Lewis Research Center

Cleveland, Ohio

Prepared for the

32nd Aerospace Sciences Meeting and Exhibit

sponsored by the American Institute of Aeronautics and Astronautics Reno, Nevada, January 10-13,1994 


\title{
EFFECT OF 'DELTA TABS' ON MIXING AND AXIS SWITCHING IN JETS FROM ASYMMETRIC NOZZLES
}

by

\author{
K. B. M. Q. Zaman \\ NASA Lewis Research Center \\ Cleveland, OH 44135
}

\begin{abstract}
The effect of delta tabs on mixing and the phenomenon of axis switching in free air jets from various asymmetric nozzles was studied experimentally. Flow visualization and Pitot probe surveys were carried out with a set of small nozzles $(D=1.47 \mathrm{~cm})$ at a jet Mach number, $M_{j}$ $=1.63$. Hot wire measurements for streamwise vorticity were carried out with larger nozzles (D) $=6.35 \mathrm{~cm}$ ) at $\mathrm{M}_{\mathrm{j}}=0.31$. Jet mixing with the asymmetric nozzles, as indicated by the mass fluxes downstream, was found to be higher than that produced by a circular nozzle. The circular nozzle with four delta tabs, however, produced fluxes much higher than that produced by the asymmetric nozzles themselves or by most of the tab configurations tried with them. Even higher fluxes could be obtained with only a few cases, e.g., with a 3:1 rectangular nozzle with two large delta tabs placed on the narrow edges. In this case the jet 'fanned out' at a large angle after going through one axis switch. The axis switching could be either stopped or augmented with suitable choice of the tab configurations. Two mechanisms are identified governing the phenomenon. One, as described in Ref. 12 and referred to here as the $\omega_{\theta}$-induced dynamics, is due to differential induced velocities of different segments of a rolled up azimuthal vortical structure.
\end{abstract}

The other is the $\omega_{x}$-induced dynamics due to the induced velocities of streamwise vortex pairs in the flow. While the former dynamics are responsible for rapid axis switching in periodically forced jets, the effect of the tabs is governed mainly by the latter. It is inferred that both dynamics are active in a natural asymmetric jet issuing from a nozzle having an upstream contraction. The tendency for axis switching caused by the $\omega_{\theta}$-induced dynamics is resisted by the $\omega_{x}{ }^{-}$ induced dynamics, leading to a delayed or no switchover in that case. In jets from orifices and in screeching jets, the $\omega_{\theta}$-induced dynamics dominate causing a faster switchover.

\section{Introduction}

In an effort to increase mixing in free jets, a 'passive control' method, utilizing vortex generators in the form of 'tabs', has been under investigation for the past several years. ${ }^{1-6} \mathrm{~A}$ notable earlier study on the topic is that of Bradbury and Khadem. ${ }^{7}$ Tabs are protrusions projecting into the flow at the jet nozzle exit. That tabs eliminate screech noise from supersonic jets has been known for a long time, ${ }^{8}$ and there have been further recent studies on the effect of tabs on jet noise. ${ }^{9,10}$ However, the emphasis of the continuing investigation at NASA Lewis Reseach Center with regards to the topic has been on 
mixing enhancement and on obtaining a clearer understanding of the underlying mechanisms.

A parametric study of the tab geometry, to optimize the effect for a given area blockage, yielded the 'delta tab' configuration which is to be explained in the following. Earlier results obtained with delta tabs on supersonic and subsonic axisymmetric jets have been summarized in Ref. 6. These results clearly demonstrate an enormous distortion of the jet cross section produced by the delta tabs. Some combinations of tabs significantly increase the mixing and entrainment, much more than that achieved by any other method tried previously, such as acoustic excitation. Also discussed in Ref. 6 is the generation mechanism of streamwise vorticity in the flow. Two sources are identified: one is due to an upstream pressure hill produced by the tab, and the other is due to vortex filaments shed from the sides of the tab and reoriented downstream by the mean shear of the mixing layer. While the second source is somewhat similar to the mechanism producing the well known trailing vortices in the flow over a delta wing, it is shown that the first source is the dominant one in the present flow.

Presently the investigation has been expanded to study the effect of delta tabs on jets from asymmetric nozzles. Jets from such nozzles, for example, a 3:1 elliptic one, have been observed before to mix more efficiently compared to the axisymmetric case. ${ }^{11-14}$ There have been a number of prior studies on jets from rectangular nozzles, ${ }^{15-20}$ some of which also indicated more efficient mixing compared to the circular case. However, based on the available data a clear and direct comparison of the mixing characteristics of jets from the various nozzles has been difficult. Part of the difficulty lies in the fact that mixing or spreading of a jet can be defined in a number of ways, and different studies have used different parameters for that purpose. Many of the parameters, such as streamwise variation of the jet half-velocity-width or the centerline velocity, would only provide a partial description, and could even be misleading, in the case of the asymmetric nozzles. Perhaps, a fool-proof description of the overall mixing would be given by the mass flux variation with streamwise distance. But obtaining such data is difficult, prone to errors, and time consuming, which is why only a few previous researchers carried out such measurements. Another serious problem in the direct comparison of jet mixing for nozzles of different geometries stems from differences in the operating conditions in the previous studies, especially in Mach number. It is well known that mixing layer spreading reduces with increasing compressiblity at higher Mach number. Therefore, a comparison of mixing with different nozzles should be appropriate only when done at exactly the same Mach number and preferably with other operating conditions, such as Reynolds number and initial boundary layer state, kept the same as well.

Thus, it was felt that a systematic experimental study was called for first to compare the mixing characteristics in jets from nozzles of different geometries without tabs. This has been one of the objectives of the present investigation. The effect of delta tabs on the jets from these nozzles has also been explored. During these explorations it has been found that delta tabs not only affect the mixing but also the phenomenon of axis switching. Axis switching or switchover is common in asymmetric jets. It is a phenomenon in which the jet cross section contracts in the direction of the major axis and expands along the minor axis, so that after a certain downstream distance the two axes have interchanged. Refer- 
ence 16 provides data on axis switch location as a function of nozzle aspect ratio. Reference 12 addresses a possible mechanism of the phenomenon which will be discussed further in the text. An investigation of axis switching and jet mixing, as affected by the delta tabs, has been the second objective of the present study. The results are discussed in the following.

\section{Experimental Facility and Method}

The experiments were carried out in two different jet facilities. Most of the supersonic jet experiments were done in a smaller facility which has been described in detail before. ${ }^{2,6} \mathrm{Com}$ pressed air supplied through one end of a $11 \mathrm{~cm}$ diameter plenum chamber exited through a 3.81 cm diameter 'nipple' on the opposite end. Nozzles of different exit geometry could be attached to the nipple. The larger facility, with a $76 \mathrm{~cm}$ diameter plenum chamber, has also been described previously. ${ }^{21,22}$ Detailed time averaged properties and streamwise vorticity were measured in this facility at low subsonic conditions. Limited Pitot probe surveys were also conducted at supersonic conditions in this facility.

All asymmetric nozzles used in the experiment were machined from solid cylindrical blocks of aluminum by electrical discharge machining (EDM). The interior along the major and the minor axes in each case was contoured according to fourth order polynomial fits. The rest of the interior was faired smoothly without following specific equations. In all cases the flow always converged, and entered and exited approximately axially. The general interior shape of each nozzle is shown schematically in Fig. 1(a); all had 'end walls' to facilitate easy installation of the tabs at desired locations.

A triangular tab fitted to a circular nozzle is shown schematically in Fig. 1(b). The specific

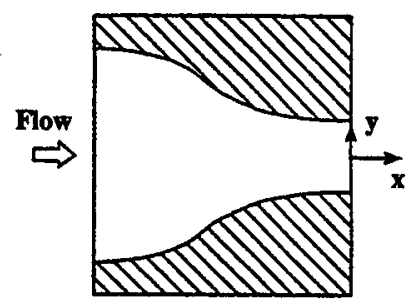

(a)

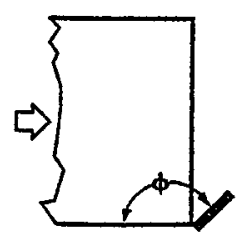

(b)
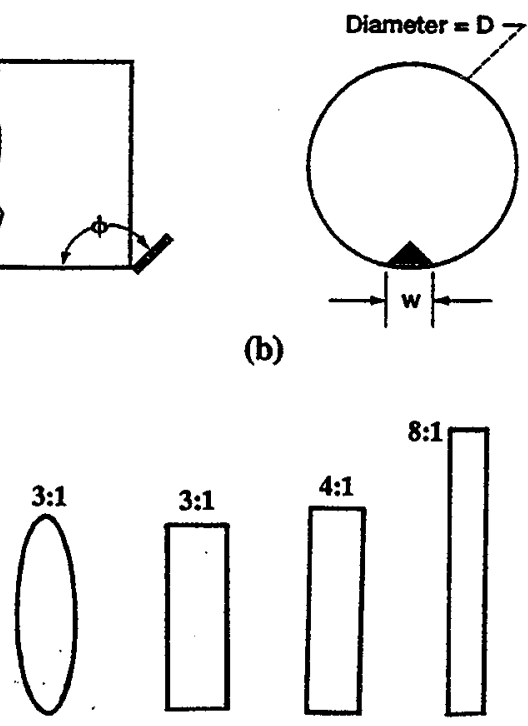

(c)

Fig. 1 Schematics of: (a) nozzle interior shape, (b) delta tab fitted to axisymmetric nozzle, and (c) exit geometries of the asymmetric nozzles.

configuration with orientation $\phi \approx 135^{\circ}$ is referred to as the 'delta tab'. The apex angle is about $90^{\circ}$, and unless otherwise stated the base width, w/D $\approx 0.28$. Even though this width is large, the dimensions translate to an area blockage of about 1.7 percent of the nozzle exit area. The delta tabs were hand sheared and bent and thus the angles and dimensions quoted are approximate.

Besides a circular one, four asymmetric nozzles were used in the smaller facility. These were: a 3:1 elliptic, and a 3:1, a 4:1 and a 8:1 rectangular, all having $\mathrm{D}=1.47 \mathrm{~cm}$. For the 
asymmetric nozzles the notation $D$ represents the equivalent diameter based on the exit area. The four asymmetric shapes are sketched in Fig. 1(c). It should be mentioned here that the circular nozzle, fabricated earlier, ${ }^{2}$ had a smaller diameter $(\mathrm{D}=1.27 \mathrm{~cm})$. Thus, for a given Mach number, the Reynolds number was somewhat smaller in the axisymmetric case. This difference was deemed insignificant in the comparison of the normalized mass fluxes. In the larger facility, a circular and a 3:1 rectangular nozzle were used; both had $\mathrm{D}=6.35 \mathrm{~cm}$.

For the supersonic jets, the notation $\mathrm{M}_{\mathrm{j}}$ is used to denote the 'fully expanded Mach number', i.e., the Mach number had the jet expanded to ambient pressure for a given plenum chamber pressure. Standard total pressure measurements, with a $0.76 \mathrm{~mm}$ (o.d.) Pitot tube, were made to obtain variations of the stagnation pressure $\left(P_{V}\right)$; note that in the supersonic regions of the flow, this pressure represents the stagnation pressure downstream of the bow shock produced by the probe itself.

Flow visualization pictures for the supersonic jets were obtained by laser sheet illumination. This was performed without any artificial seeding of the flow. The cold supersonic jet core caused natural moisture condensation in the mixing layer from the entrained ambient air. The condensed moisture particles, and therefore the mixing layer regions, were illuminated by the laser sheet. The exposure time (T) for each picture was long $\left(\mathrm{TU}_{\mathrm{j}} / \mathrm{D}>270\right)$ so that these represent averages rather than instantaneous flow fields.

Conventional hot-wire measurements were carried out to obtain the distributions of time averaged vorticity and turbulence statistics on a cross sectional plane of the jet. The measurements could be carried out only for the subsonic flow, and a nominal $\mathrm{M}_{\mathrm{j}}=0.3$ was chosen. Two $\mathrm{X}$-wire probes, one in the $\mathrm{u}-\mathrm{v}$ and the other in the $u-w$ configuration, were traversed successively over the same measurement grid locations. The distributions of the $u, v$ and $w$ components of velocity and the turbulence characteristics were obtained. The gradients of $v$ and $w$ provided the streamwise vorticity. These measurements were done in the larger jet so that the spatial resolution provided by the X-probes (TSI 1241) was reasonably good. Nevertheless, estimates based on mixing layer thickness and sensor separation in the $\mathrm{X}$-probe $(\approx 1 \mathrm{~mm})$ showed that corrections were needed for the $\mathrm{v}$ - and $\mathrm{w}$-components of velocity especially at the upstream locations. The data were corrected following a scheme similar to that used in Ref. 23.

\section{Results}

3.1 Comparative data on mixing with different nozzles: The Mach number distributions over the jet cross sectional plane, measured at $x / D=14$ for different nozzles, are shown in Fig. 2. These data were taken far enough downstream so that the flow was subsonic everywhere and the static pressure had relaxed to ambient pressure. Hence the Mach number could be calculated reliably from only the Pitot probe measurement. Also shown in Fig. 2 is the corresponding distribution for the circular jet with four delta tabs. Four delta tabs (among one, two, four and six delta tab cases) had been shown to produce the optimum increase in the mass flux for a circular nozzle. ${ }^{6}$ The large effect of the tabs on the jet spread can be readily appreciated from Fig. 2 .

In each of the plots of Fig. 2, the mass flux obtained by integrating the data, normalized by the exit mass flux, has been indicated by the number in parentheses. The maximum Mach number measured at each station is also indi 

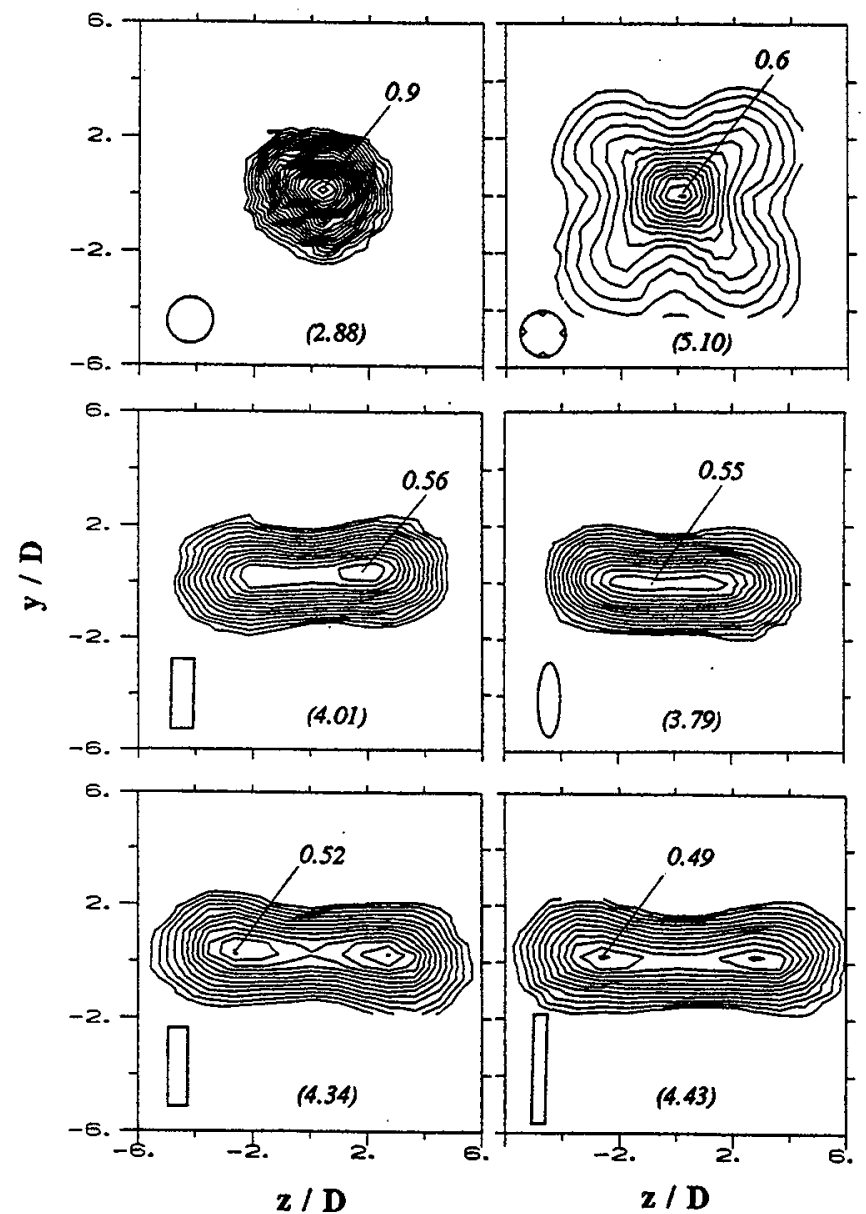

Fig. 2 Mach number contours for indicated nozzles; $x / D$ $=14, M_{\mathrm{j}}=1.63$. Contour levels start at 0.04 and the interval is 0.04; numbers in parentheses represent normalized mass fluxes.

cated. An inspection of these numbers should indicate that the fluxes for all the asymmetric jets are larger than that for the axisymmetric case. However, none of them exceeds the case with the four delta tabs. Also, as expected, the maximum Mach number is generally less when the flux is high and vice versa. For all four asymmetric cases in Fig. 2, it is also observed that the jet major axis which started aligned vertically has switched and become horizontal by $x / D=14$.

The mass flux variations, obtained from sets of data as in Fig. 2, are shown in Fig. 3. In

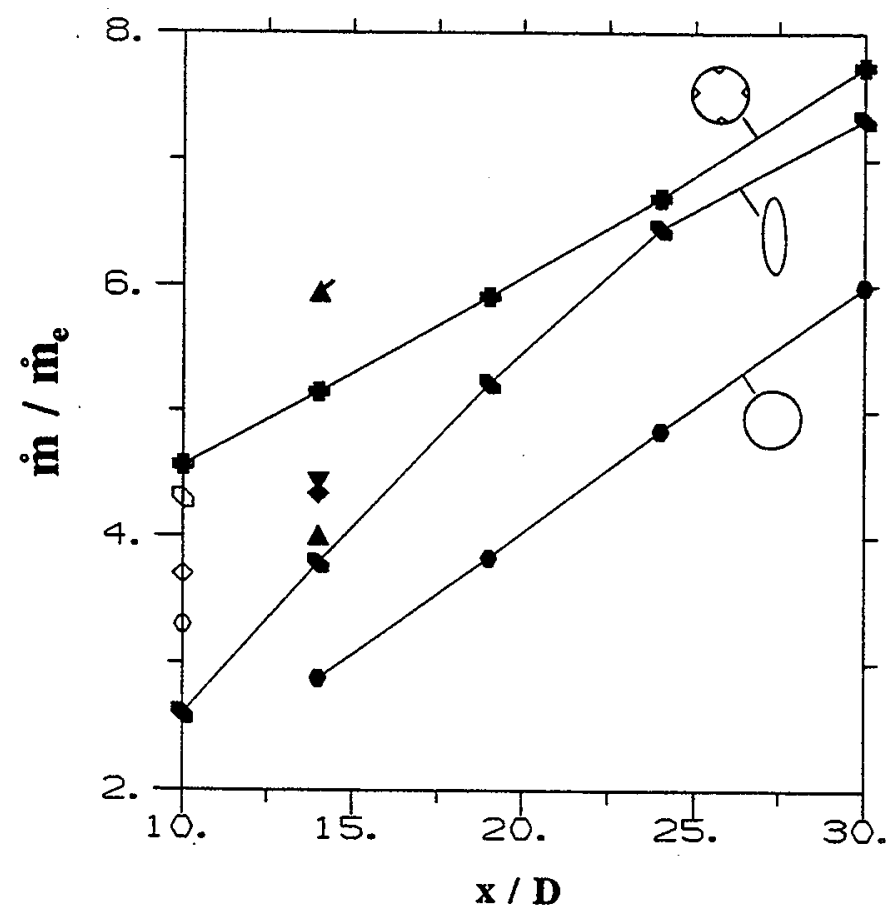

Fig. 3 Streamwise variation of normalized mass flux for indicated cases; $M_{j}=1.63$. Other data points at $x / D=$ 14 are for: $\iota, 3: 1$ rectangular, $\bullet, 4: 1$ rectangular $\nabla$, 8:1 rectangular nozzles, and $x, 3: 1$ rectangular nozzle with two large delta tabs. Open data points at $x / D=10$ are for: $0,3: 1$ elliptic jet at $M_{j}=0.06$ (Ref. 11); 0 , unexcited and $\diamond$, acoustically excited circular jet at $\mathbf{M}_{\text {; }}$ - 0.1 (Ref. 24).

the Pitot tube measurements, there are some errors due to flow angularity and turbulence especially at the edges of the jet. Since the integrated flux values are sensitive to small errors on the edges, the flux data should be considered as nominal. However, the relative trends in the data should be valid. Also, sufficient averaging time was allowed and care was taken so that the repeatability of the data was good. A few data points were double checked to repeat practically within the data symbol heights in Fig. 3.

The data of Figs. 2 and 3 were obtained with the small nozzles. When setting up the experiment in the large jet facility it was intended that some of these data be repeated with the large 
nozzles. However, although the larger facility could deliver much higher flow rates at higher pressures, the experiments could not be run at $M_{j}$ $=1.63$ because of unacceptably high noise levels in the work environment. The noise levels were, however, within acceptable range when four delta tabs were used with the circular nozzle. For that case, in spite of appreciable recirculation velocities (about $4 \mathrm{~kg} / \mathrm{sec}$ in a $6 \mathrm{mx} 6 \mathrm{mx} 12 \mathrm{~m}$ test cell), the Pitot probe surveys were done at $x / D=14$. A very similar M-distribution as the corresponding data in Fig. 2, with a somewhat smaller normalized mass flux of 4.8 , was obtained. The difference in the flux data, however, was small. Thus, the four delta tab case data is deemed independent of nozzle size and Reynolds number. However, it is emphasized that the data of Fig. 3 apply to underexpanded flows from convergent nozzles at $\mathrm{M}_{\mathrm{j}}=1.63$; the amplitudes are likely to change for other states of the flow and especially for other values of $M_{j}$.

It should also be mentioned here that while calculating the exit mass flux for the tab cases, the area blockage due to the tabs has been taken into account. ${ }^{6}$ If this were not done, the data points for the four delta tab case would be about six percent less everywhere; nevertheless, the conclusions regarding the relative increase in mixing by the tabs would remain unchanged.

The elliptic jet case in Fig. 3 exhibit fluxes in between the baseline axisymmetric and the four delta tab cases. The spreading rate is faster upstream but slower farther downstream. Clearly, the elliptic jet flow field is still evolving within the measurement range. In other words, the flow field represents, in the terminology of Ref. 15, the 'characteristic decay region' and has not reached the 'axisymmetric decay region'. Data for the rectangular jets were obtained only for $x / D=14$. Unfortunately, these data sets covering the entire $\mathrm{x}$-range could not be completed in time to be included in Fig. 3. It is likely that the rectangular jets would also exhibit evolving flow fields as with the elliptic jet. It is also probable that, at the farthest $x$-stations, some of these cases could exhibit fluxes larger than that for the four delta tab case. However, at least in the upstream regions, as with the elliptic jet, the fluxes for the rectangular jets are higher than that for the circular jet case but none exceeds that for the four delta tab case.

The three open data points on the left margin of Fig. 3 are for subsonic jets taken from the references indicated in the figure caption. These include a data point extrapolated from the results for a 3:1 elliptic jet, ${ }^{11}$ and data for unexcited and acoustically excited circular jets. ${ }^{24}$ An inspection reveals that the flux for a subsonic jet is higher than that for the corresponding supersonic counterpart; this is expected because mixing layer spreading reduces with increased compressibility. It is also clear that the elliptic jet flux even for the subsonic case does not exceed that of the 4 delta tab case. Comparison of the data from Ref. 23 also show that the increase in the flux due to the 'preferred mode' acoustic excitation is far less than that achieved by the delta tabs. Thus, the increase in the axisymmetric jet mixing caused by the delta tabs should be considered as quite significant.

Various tab configurations were tried with the asymmetric jets. As the number of combinations are numerous, the effort should be considered as limited. The mixing characteristics for some of the tab cases with the asymmetric jets will be discussed in the following. It should suffice to state here that most of these cases resulted in fluxes lower than that achieved by four delta tabs with the circular jet. For example, two delta tabs placed on the narrow ends of the 
(a)

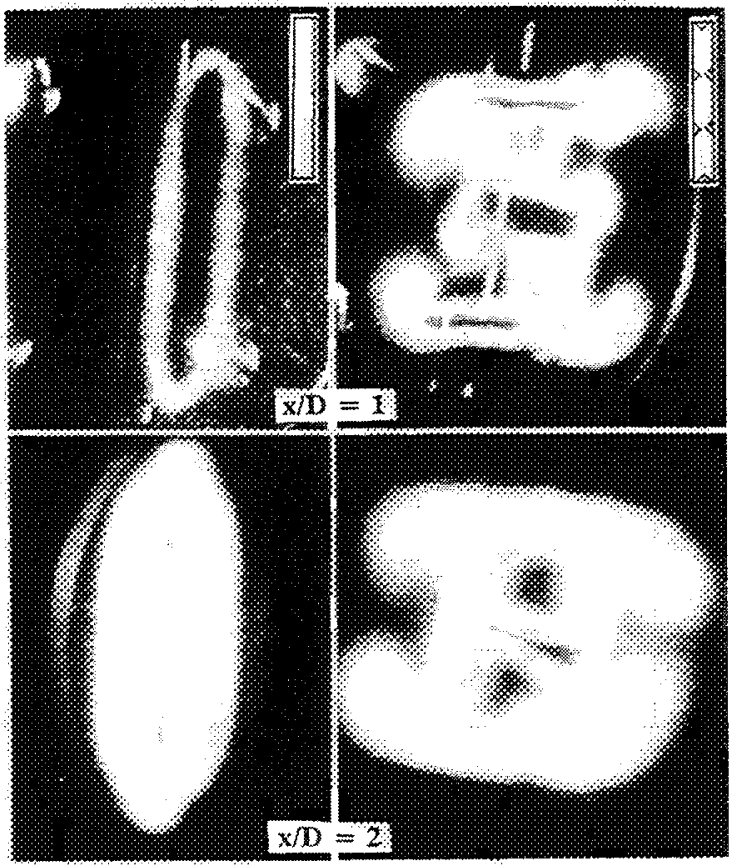

Fig. 4(a,b)

3:1 elliptic jet produced a normalized flux of 4.53 at $x / D=14$, corresponding data with two delta tabs placed on the longer edges was 3.1, while the no tab case flux was 3.79. Note that the latter tab case actually decreased the flux. Only a few configurations so far yielded fluxes significantly higher than that for the circular jet with four delta tabs. One such case is the 3:1 rectangular jet with two large delta tabs placed on the narrow edges of the nozzle. The corresponding data at $x / D=14$ is shown by the triangular data point with a tick mark (Fig. 3). The tab dimension and the flow field for this case is discussed further in §3.3.

\subsection{Axis switching and its mechanism: As stated} before, both mixing and the phenomenon of axis switching for the asymmetric jets were found to be sensitive to the number and placement of the (c)

(d)

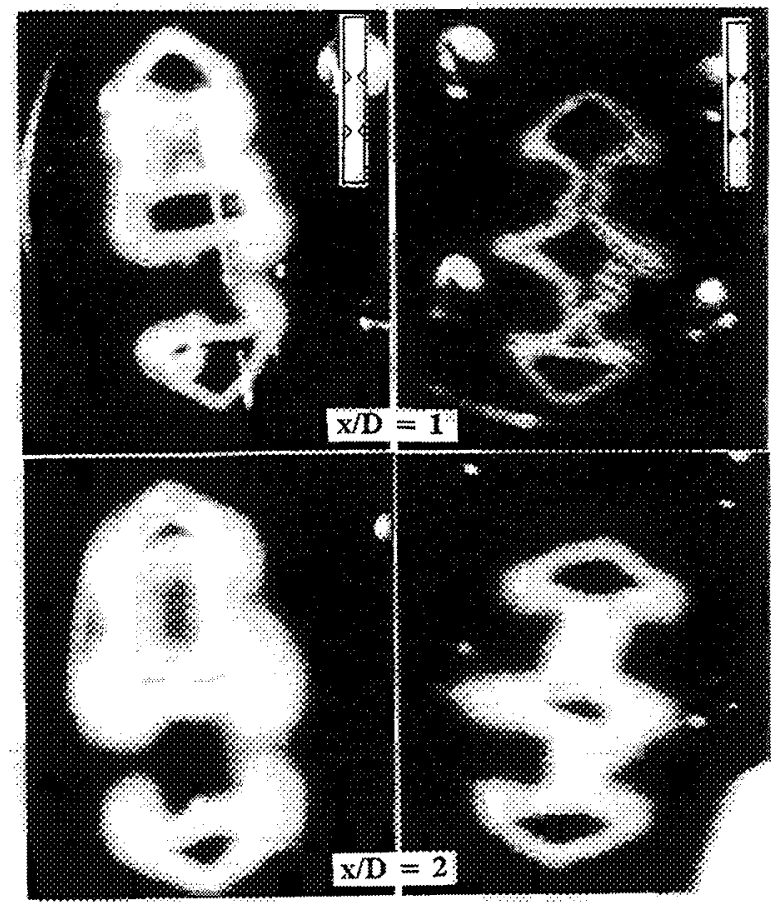

Fig. 4 Laser sheet illuminated cross section of 8:1 rectangular jet $(D=1.47 \mathrm{~cm})$ at $M_{\mathrm{j}}=1.63$; (a) no tab, (b) six delta tabs, (c) four delta tabs, and (d) four triangular tabs with $\phi=45^{\circ}$ (Fig. 1).

delta tabs. These effects were pursued in some detail with the 8:1 rectangular jet at $\mathrm{M}_{\mathrm{j}}=1.63$. Fig. 4 shows laser sheet illuminated cross sections of the jet, at $x / D=1$ and 2 , for three tab configurations compared to the no tab case. The tab configurations are shown by the insets and explained in the figure caption. The bright areas in the pictures represent the mixing layer region. ${ }^{2}$ The enormous distortions of the mixing layer produced by the delta tabs should be quite obvious. Even though the fluxes farther downstream are not increased significantly, as will be shown with the next figure, the large spreading caused by the tabs immediately downstream of the nozzle could be attractive in some applications. One also notes that suitably placed delta 
(a)
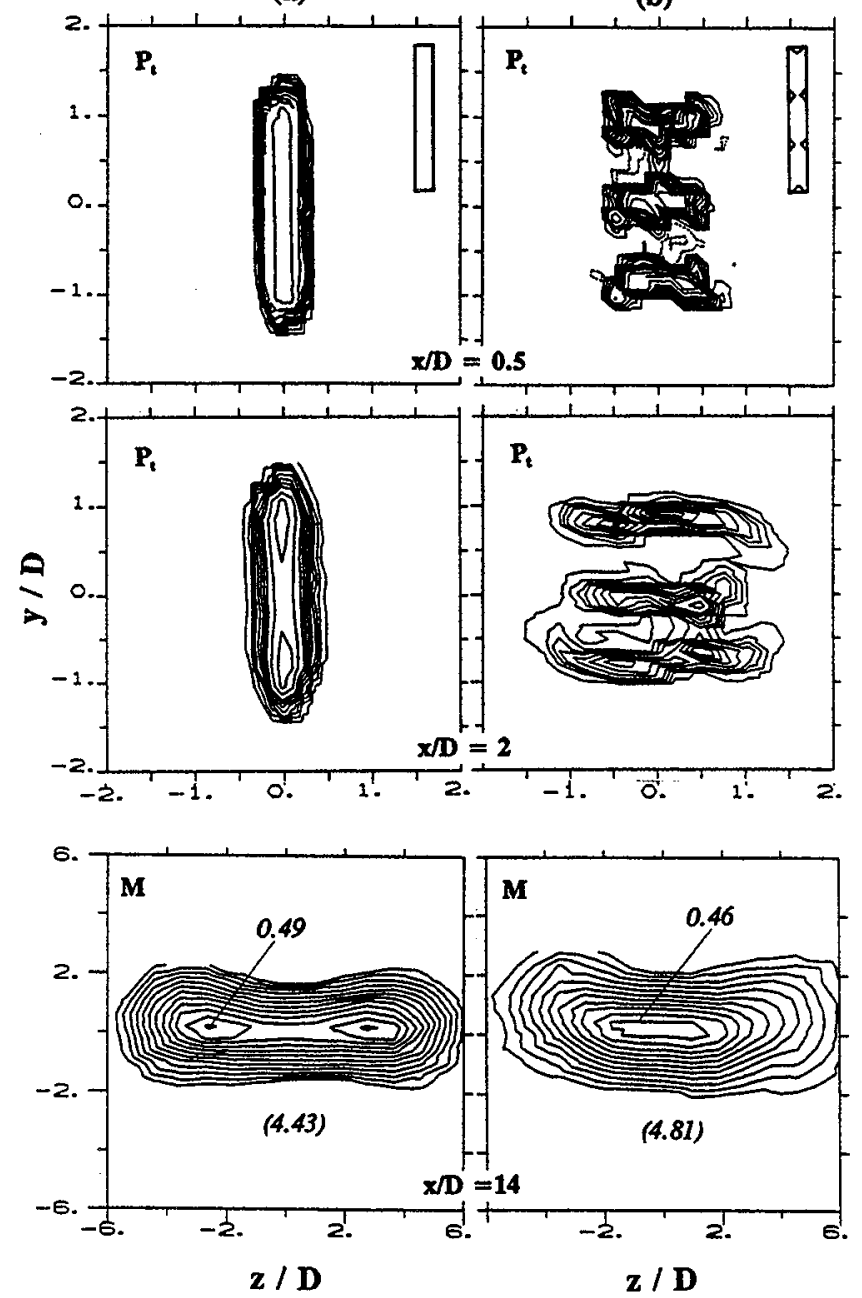

Fig. 5(a,b)

tabs within a simple primary nozzle can produce effects similar to that achieved by more complex nozzles, such as a lobed mixer nozzle.

The evolution of the flow fields for the tab cases of Fig. 4 is shown in Fig. 5. For each case data are presented for three streamwise locations. At the upstream locations where the flow is supersonic contours of total pressure are shown. At the downstream location $(x / D=14)$ the Mach number distributions as in Fig. 2 are shown. Even though there are some errors due to probe interference, the $P_{t}$ data confirm the (c)
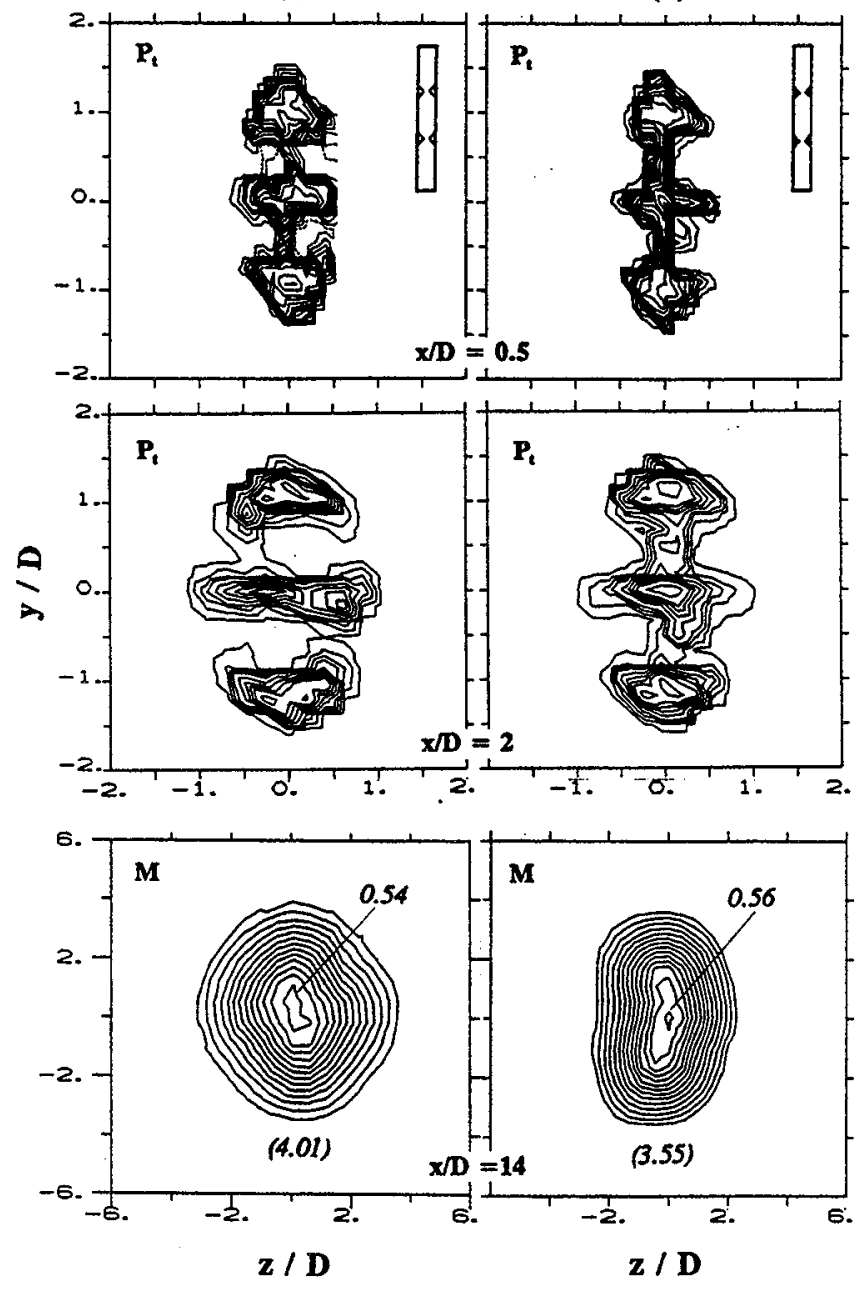

Fig. 5 Contours of total pressure $(P)$ and Mach number (M) at indicated $x / D$; sets of data in columns (a)-(d) represent corresponding tab cases of Fig. 4.

dramatic deformations of the jet cross section seen in Fig. 4. By 14D in case (b), however, the jet has regained its original shape with only about 9 percent increase in the mass flux compared to the no-tab case. Axis switching has also taken place as in the no-tab case. In case (c), the jet cross section has become round, whereas in case (d) the major axis has remained approximately vertical. The numbers in parentheses should also reveal that the tab configurations of (c) and (d) 
have reduced the mass flux at $x / D=14$ from what it was for the no tab case. As stated before a similar effect was also observed with the elliptic jet.

It has been shown before that each delta tab produces a pair of streamwise vortices. ${ }^{6,21}$ The interaction of those vortices with the azimuthal vorticity in the mixing layer, and with the naturally occurring streamwise vortices in these flows, ${ }^{20}$ is believed to be at the heart of the observed effects. This was explored further by hot wire measurements in the large jet facility.

First, a comparison of Mach number contours, obtained with the Pitot probe in the large jet, is shown in Fig. 6 for $M_{j}=0.31$ and 1.21. It is an example showing that the effect of the delta tabs on the axis switching phenomenon, for most of the cases studied, is essentially the same in both supersonic and subsonic flow regimes. In both cases of Fig. 6 there has been an axis switch. The major axis is somewhat off from the vertical, which is also quite similar in the two cases. Thus, the effect on axis switching

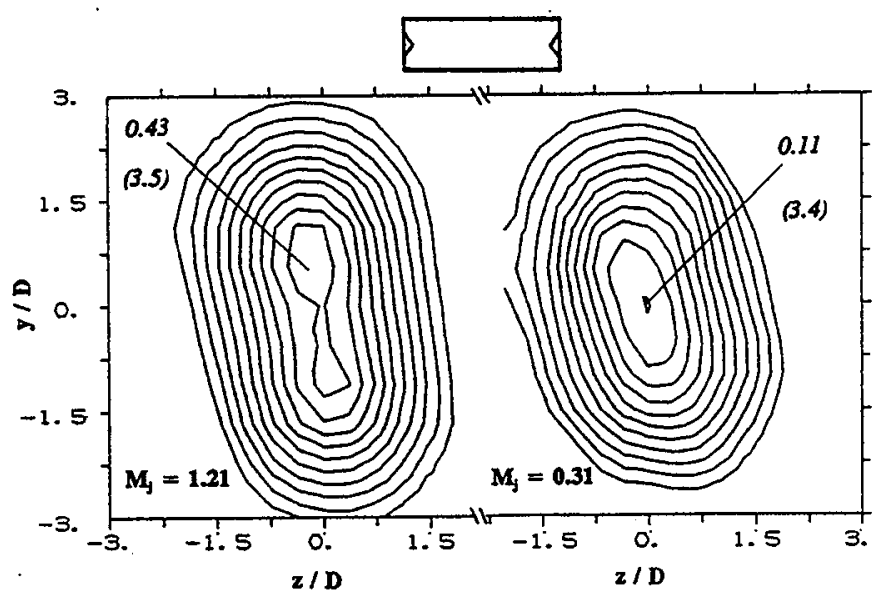

Fig. 6 M-contours for the 3:1 rectangular jet $(D=6.35$ cm) with two delta tabs for indicated $M_{j} ; x / D=14$. Contour intervals are 0.04 and 0.01 for $M_{j}=1.21$ and 0.31 cases, respectively. studied in incompressible flows and reported in the following is thought to be also representative of high Mach number flows. However, without the tabs some differences were observed between supersonic and subsonic conditions; this is discussed shortly.

The flow field for the 3:1 rectangular jet was explored in detail for two tab configurations and compared to the no tab case. Fig. 7 shows the streamwise variations of the jet half-velocitywidth measured in the major and the minor axis planes of the nozzle; $B$ represents the distance between the points where the velocity is half of the local centerline velocity. One finds that within the $\mathrm{x}$-range covered, the jet without any tabs has not gone through an axis switch. However, two delta tabs placed on the narrow edges of the nozzle have caused a rapid switchover by $\mathrm{x} / \mathrm{D}=2.5$. In comparison, two delta tabs placed on the long edges have caused the jet to continue to diverge on the major axis plane within the measurement range.

Comparison of Fig. 7 with Fig. 2 shows that the 3:1 rectangular jet without any tabs behaves differently at $\mathrm{M}_{\mathrm{j}}=0.31$ than it does at $M_{j}=1.63$. While there has been an axis switch by $x / D=14$ in the supersonic case (Fig. 2), it has not taken place at the subsonic condition (Fig. 7). It is probable that the reason for this difference is the screech occurring at the supersonic condition. (All the no tab case flows in Fig. 2 had screech; the screech frequency was $8.9,6.3,6.3,6.55$ and $8.35 \mathrm{kHz}$ for the circular, the 3:1 elliptic, and the $3: 1,4: 1$ and $8: 1$ rectangular nozzles, respectively; note the smaller diameter of the circular nozzle.)

An asymmetric jet with screech is a periodically forced flow in which the axis switching may occur by a mechanism described in Ref. 12. Forcing results in an orderly roll up 


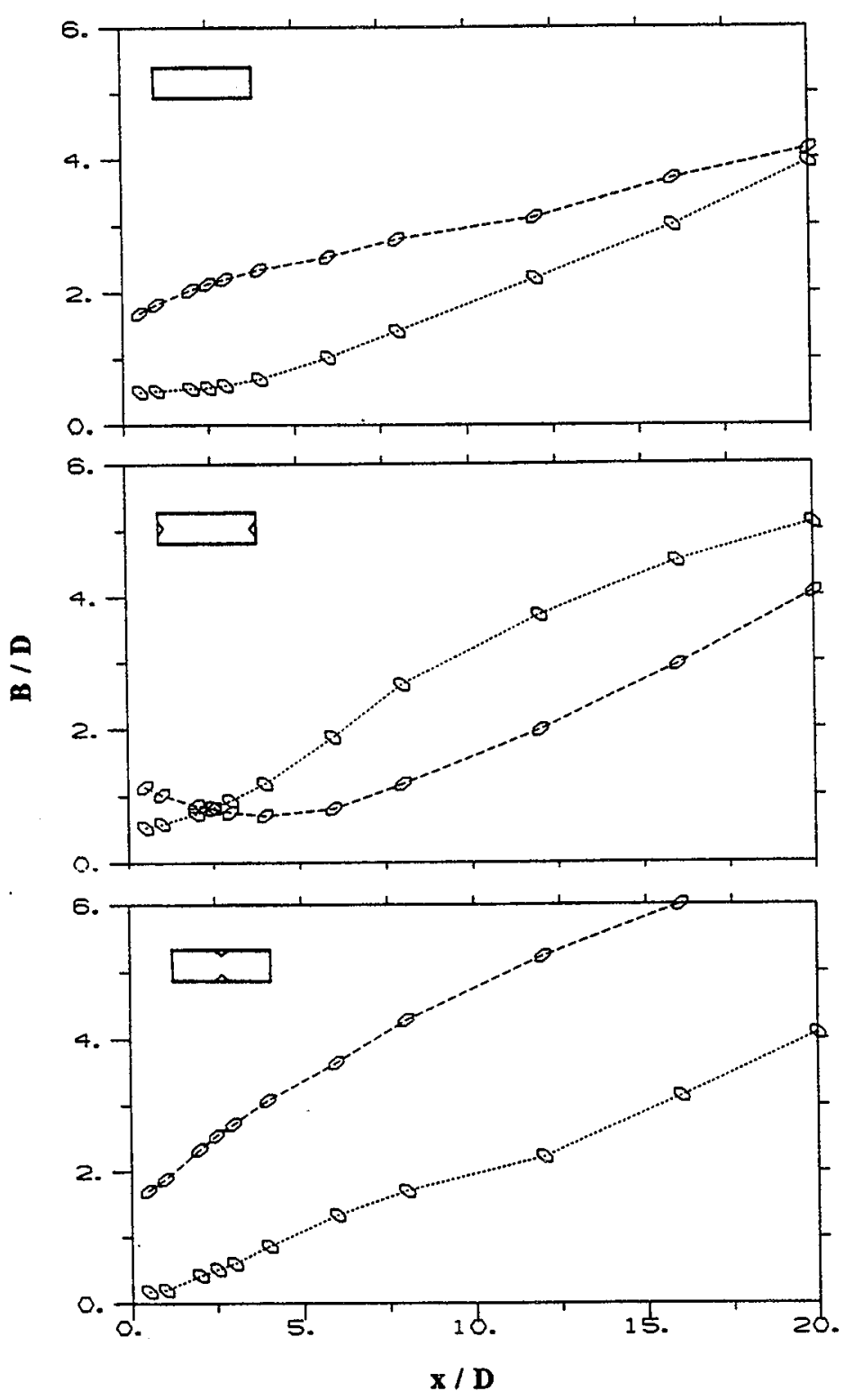

Fig. 7 Half-velocity-widths of the 3:1 rectangular jet (D $=6.35 \mathrm{~cm}$ ) at $\mathrm{M}_{\mathrm{j}}=0.31$ for indicated delta tab configurations; $O$, minor plane, $O$, major plane.

of the azimuthal vorticity. Different segments of an asymmetric rolled up vortex structure have different induced velocities attributable to differences in curvature. The segments on the ends of the major axis, having higher curvature, move forward at a faster speed relative to the segments on the ends of the minor axis. The rolled up vortex structure thus contorts as it propagates downstream; the segments on the narrow ends are brought closer together while the segments on the sides are pushed apart. This dynamic process reflects on the time average flow field and causes the switching of the axes. The process, thus, is based on the motion of the rolled up azimuthal vorticity, and for brevity, this is referred to as the $\omega_{\theta}$-induced dynamics. Such a dynamics should be active in the screeching jet, most probably explaining the fast switchover of the axes observed in Fig. 2.

The $\omega_{\theta}$-induced dynamics are also applicable to unforced asymmetric jets as there is a natural roll up of the azimuthal vorticity. However, in the unforced case there is randomness, which in a sense dilutes the effect, causing a delayed axis switching. That a forced case goes through faster axis switching compared to the corresponding unforced case has been demonstrated in Ref. 12 by the use of acoustic excitation of an elliptic jet. However, the $\omega_{\theta}$-induced dynamics do not explain the effects of the delta tabs on the axis switching. The observed effect of the tabs is believed to be due to an entirely different process involving the interaction of the streamwise vortices.

Data showing the evolution of the flow field corresponding to the three cases of Fig. 7 are presented in Fig. 8. Contours of longitudinal velocity are shown on the left column and the corresponding contours of streamwise vorticity are shown side by side on the right column. Data are shown for five streamwise locations of $x / D=$ $1,2,4,8$ and 16. In order to provide a clearer picture of the distributions, only smoothed vorticity data are presented. The smoothing reduced the peak levels somewhat. However, representative peak values of $\omega_{x}$ as measured originally are indicated in the figures. (While longitudinal 
(a)
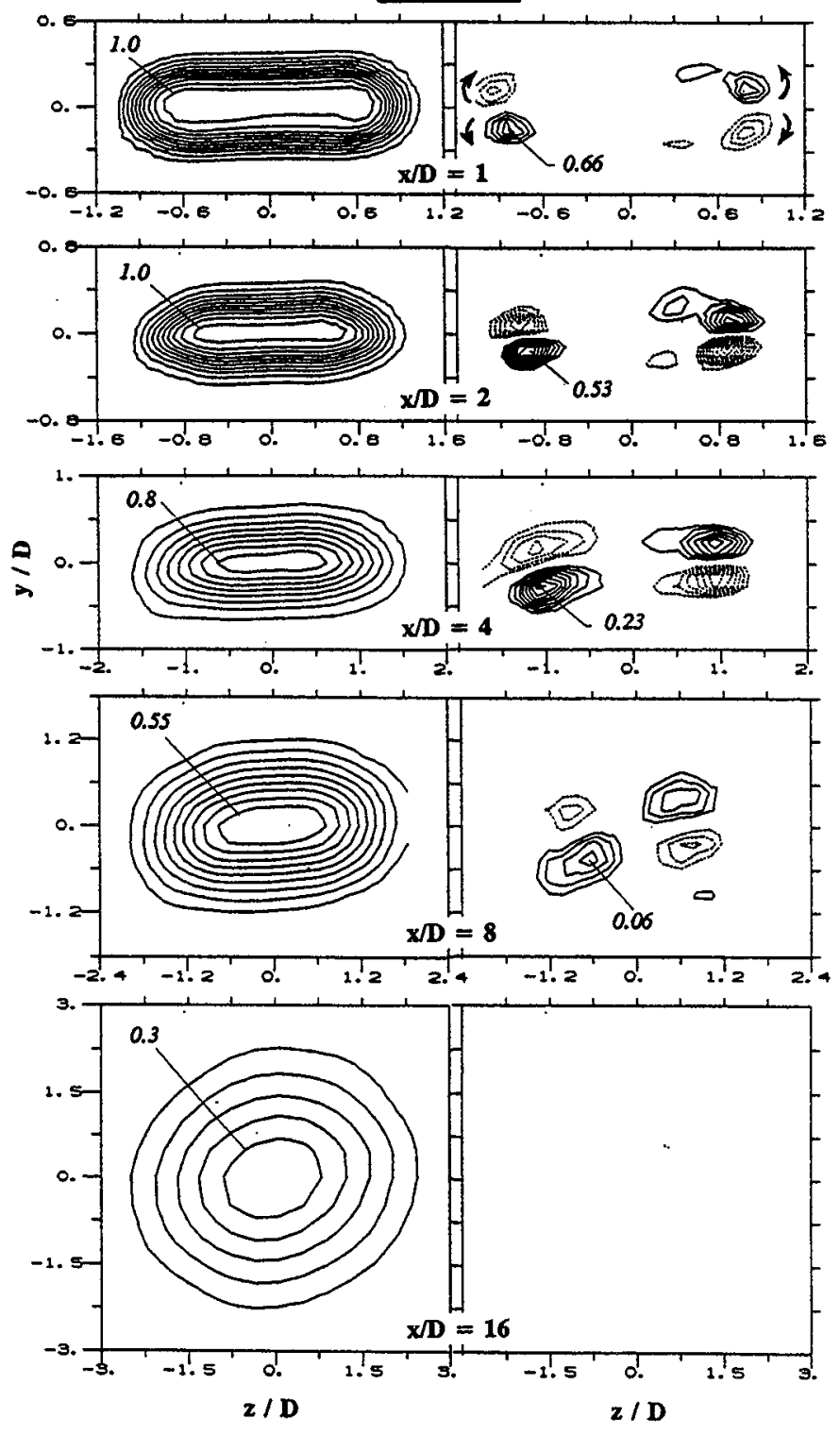

Fig. 8(a) (b)
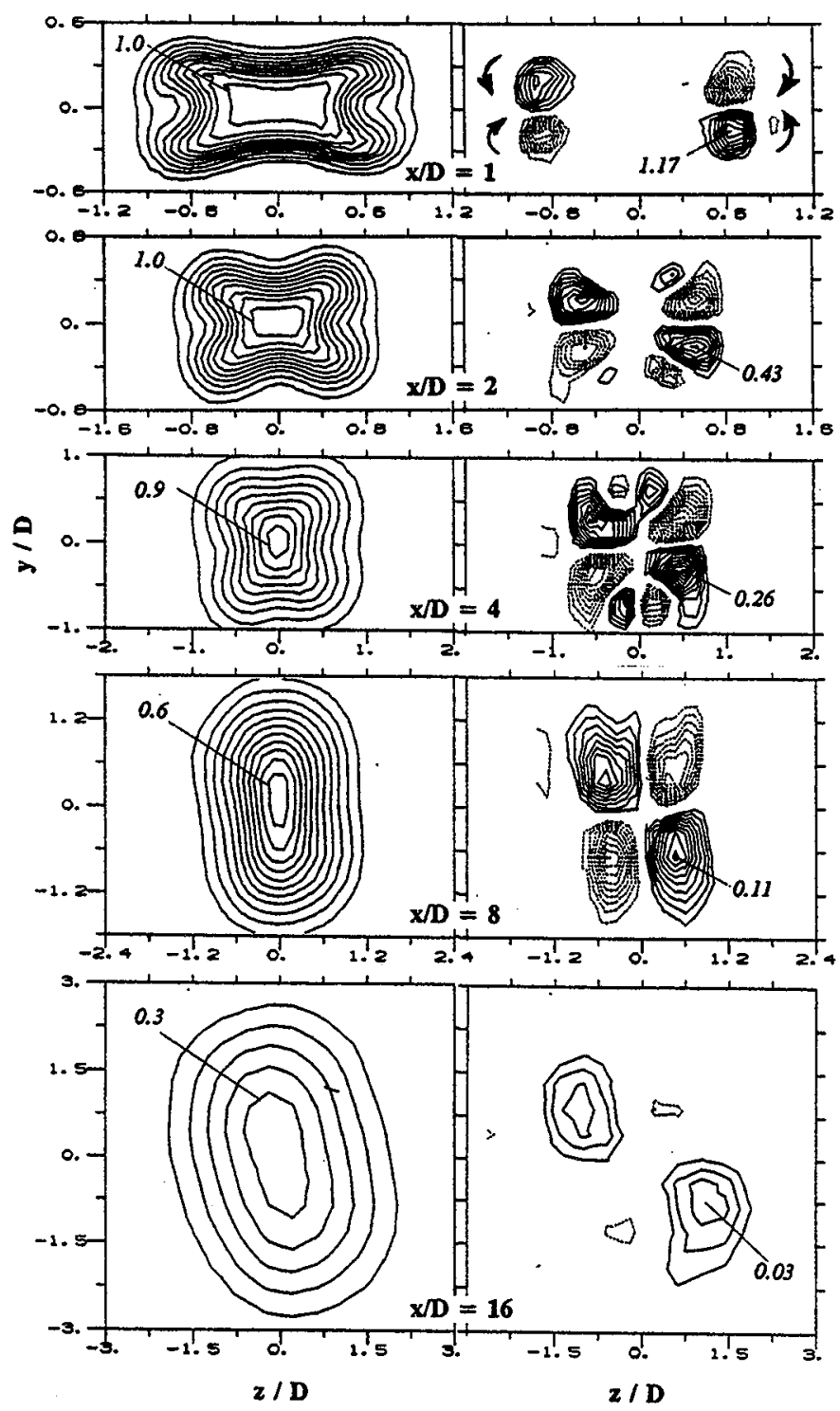

Fig. 8(b) velocity and vorticity data are shown in Fig. 8, corresponding data on the other components of velocity, turbulence and Reynolds stresses were also acquired simultaneously. These are not shown for brevity.)

In Fig. 8(a), the velocity contours show that the jet has attained approximately a round shape by $16 \mathrm{D}$. The corresponding vorticity data show the emergence of streamwise vortices from each corner of the nozzle even in the no tab case. Similar time averaged vorticity measurements for a square nozzle were reported by Quinn. ${ }^{20}$ Quinn observed a pair of counter-rotating streamwise vortices originating from each corner of the nozzle. With the present 3:1 rectangular nozzle only one vortex from each corner is seen to 
dominate by the distance of the first measurement station. Two vortices originating from the corners on a narrow edge of the nozzle are seen to form a counter-rotating pair. The sense of rotation, shown by the arrows $(x / D=1)$, is such that there is an ejection of jet core fluid into the ambient by each pair. The pair of vortices forming on each side of the square nozzle in Quinn's experiment also had the same sense of rotation. The vortices are seen to lose their strength with increasing downstream distance, and by 16D the amplitudes are below the measurement noise level.

The main source of streamwise vorticity in the present flow is likely to be the lateral pressure gradients skewing the boundary layer inside the non-circular nozzle (Prandtl's first kind of secondary flow). ${ }^{25}$ It is interesting to note here that in an experiment with flow through a duct, transitioning from circular to rectangular cross section, Davis ${ }^{26}$ also observed one dominant streamwise vortex emerging from each corner. A subsequent computational study corroborated well with the experimental results. ${ }^{27}$ However, the sense of rotation of the vortices in this flow was opposite to that observed in the present flow. The cross sectional area of the transition duct studied in Refs. 26 and 27 enlarged by $15 \%$ before reducing back to the inlet value. Reference 28 , on the other hand, reported experimental results from a very similar duct, except the cross sectional area was held constant. The sign of the streamwise vortices measured in the latter work was the same as that found in the present study! (The nozzle in the present study transitions from $13.3 \mathrm{~cm}$ diameter circular section to the $3.66 \mathrm{~cm}$ $x 11 \mathrm{~cm}$ rectangular section in a length of 15.2 $\mathrm{cm})$. Thus, it is apparent that the streamwise vorticity distribution obtained at the nozzle exit is extremely sensitive to the upstream geometry of (c)
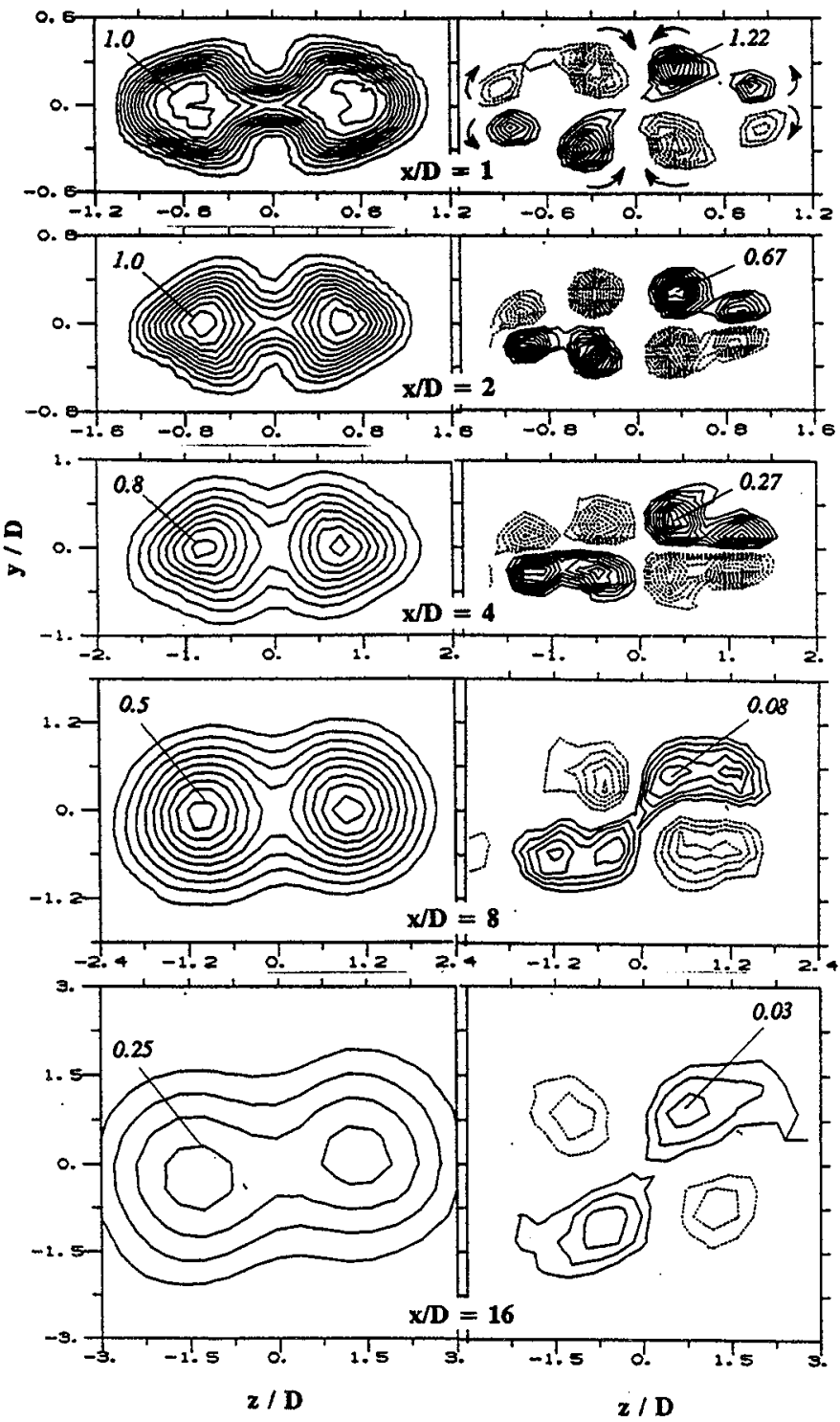

Fig. 8 Contours of $U / U_{j}$ (left column) and $\omega_{x} D / U_{j}$ (right column); 3:1 rectangular jet $(D=6.35 \mathrm{~cm}), M_{j}=0.31$. $U / U_{j}$ contour interval is 0.1 for $x / D \leq 4$ and 0.05 for $x / D$ >4. The $\omega_{x} \mathrm{D} / \mathrm{U}_{\mathrm{j}}$ contour intervals are $0.08,0.04,0.02$, 0.01 and 0.005 for $x / D=1,2,4,8$ and 16, respectively. (a) no tab, (b) two delta tabs on ends, and (c) two delta tabs on sides.

the nozzle. However, a difference in the initial $\omega_{\mathrm{x}}$-distribution can enormously impact the subsequent evolution and axis switching of the free jet, as elaborated in the following. 
Consider the vortex pairs at the upstream locations in Fig. 8(a). From elementary vortex dynamics, it should be easy to see that the induced velocities will cause each pair to move away from the jet centerline. ${ }^{29}$ Each pair will also eject jet core fluid in the same direction. Thus, the action of the two pairs of streamwise vortices would be to elongate the jet cross section in the direction of the major axis. As with the dynamics due to azimuthal vorticity, ${ }^{12}$ the effect of the streamwise vortex pairs is referred to as the $\omega_{x}$-induced dynamics. The $\omega_{\theta}$-induced dynamics, also operative in the no tab case (Fig. $8 a)$, would have a reverse influence tending to contract the jet cross section in the direction of the major axis. Thus, the jet cross section remains elongated in the major axis plane until the strength of the streamwise vortices diminishes sufficiently through turbulent diffusion. A dominant effect of $\omega_{\mathrm{x}}$-induced dynamics upstream and a persistent reverse effect of the $\omega_{\theta}$-induced dynamics reconcile the trends for the no tab case.

The $\omega_{x}$-induced dynamics should also explain the axis switching in the case of Fig. 8(b) and the no switching in the case of Fig. 8(c). For the case with two delta tabs placed on the narrow edges in Fig. 8(b), two pairs of streamwise vortices are generated which, as expected, ${ }^{6}$ have an opposite sense of rotation compared to the pairs seen in the no tab case. Here, each pair ingests ambient fluid and forces that into the core of the jet. Note also that the delta tabs overwhelm the naturally occurring vortices, which if present, are submerged below the lowest contour level at $\mathrm{x} / \mathrm{D}=1$. However, two additional pairs of vortices are seen at $x / D=2$ and 4 . It is not clear, but these could be remnants of the naturally occurring vortices gathering strength because of the contraction of the flow in the direction of the major axis. Also, in some of these data, especially at $x / D=16$, the negative vorticity amplitudes are measured to be smaller than the corresponding positive vorticity amplitudes. This would imply a net circulation in the $y-z$ plane. However, the amplitudes at $x / D=16$ are very small, and it is not clear if the discrepancy is due to measurement errors or indeed due to a small swirl introduced by deviations in the geometries of the delta tabs.

In any case, the induced velocities of the vortex pairs in Fig. 8(b) would cause each pair to move towards the jet centerline. Ambient fluid is ingested by each pair and forced into the core. The net effect is a contraction of the jet cross section in the major axis plane with a resultant elongation in the minor axis plane. This should explain the rapid axis switchover in the case of Fig. 8(b).

When two delta tabs are placed on the long edges of the nozzle (Fig. 8c), two relatively strong pairs of vortices are produced by the tabs in addition to the naturally occurring vortices. The sense of rotation of these vortices is indicated by the arrows on the plot for $x / D=1$. Here, the proximity of the vortices having like signs cause them to eventually amalgamate. The amalgamation is complete by $x / D=8$, where the four pairs of streamwise vortices have yielded two pairs. It should also be easy to see that the induced velocities of the vortex pairs in this case are similar to that in the natural case (Fig. 8a), i.e., all four pairs move away from the jet centerline in the direction of the major axis. The jet cross section thus elongates in the major axis plane. In fact, as the delta tabs in this case augment the naturally occurring vortices, the pull in the direction of the major axis is so much that the jet cross section is essentially bifurcated.

The $\omega_{x}$-induced dynamics should also explain the $45^{\circ}$ rotation of the jet cross section 
observed a few diameters downstream from the square nozzle in Ref. 20. The four pairs of streamwise vortices would tend to pull away from the axis of the jet causing such a deformation. A similar $45^{\circ}$ rotation was also observed for the cross section of a periodically forced square jet in the computational study of Ref. 19. It is not clear but the $\omega_{\theta}$-induced dynamics were likely to be mainly responsible for the rotation in this case.

Reference 30 had reported an interesting set of data on flow from small aspect ratio slot nozzles. In one case, the nozzle was essentially an orifice and the jet from it went through a rapid axis switchover by $x / D=2$. However, when a conical contraction was added leading to the nozzle exit, the resulting jet was diverging in

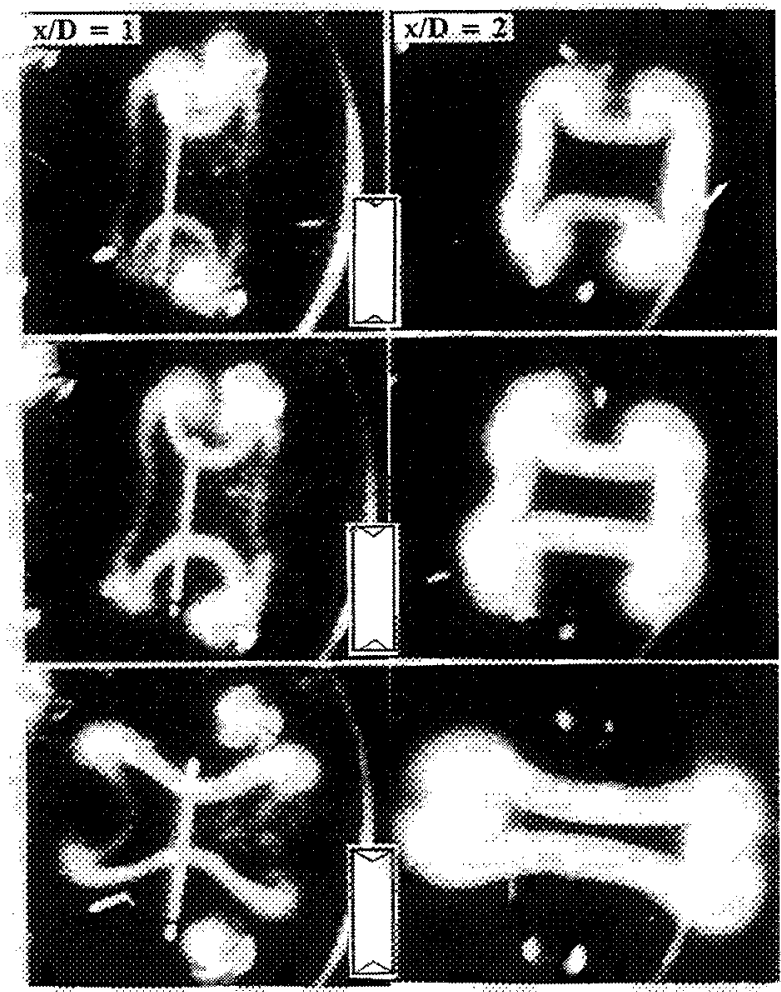

Fig. 9 Laser sheet illuminated cross section of 3:1 rectangular jet $(D=1.47 \mathrm{~cm})$ with delta tabs on ends; $M_{j}$ $=$ 1.63. Pairs of pictures from top are for tab width $w$ (Fig. 1) of 0.28D, 0.38D and 0.53D, respectively. the major axis plane within the measurement range of $x / D=10$. Such a trend corroborates the observation in Ref. 16 in which the axis switch over location was observed to be, "...further downstream for a jet exiting from a long channel than for a jet exiting from an orifice". Reference 12 also confirmed the difference in the data trend between orifice nozzles and nozzles with contraction, and provided an explanation based on the $\omega_{\theta}$-induced dynamics. In the orifice nozzles the thin initial boundary layer produces vortical structures with thin cores which would have stronger azimuthal variation in the induced velocity, and hence earlier switching of axes. However, it is also probable that in the orifice case, the flow does not produce appreciable streamwise vortices. Thus, the $\omega_{\theta}$-induced dynamics dictate a faster axis switchover. In the long channel or the contraction case, it is possible that streamwise vortices from the corners, as in the present case of Fig. 8(a), are produced. The $\omega_{x}-$ induced dynamics, resisting the axis switch, together with the $\omega_{\theta}$-induced dynamics would thus be responsible for the delayed axis switch or no switch in those cases.

3.3 A case of large mixing increase: During the investigation with the small 3:1 rectangular jet it was observed that remarkably more jet spreading could be achieved if larger size delta tabs were placed on the narrow edges of the nozzle. This was further explored. Flow visualization pictures as in Fig. 4 are shown in Fig. 9. Two delta tabs with varying widths $(w)$ are used. The delta tab width in the top pair of pictures is the same as that used for the data shown in the preceding (Figs. 6, 7 and 8). Here the ratio $\mathrm{w} / \mathrm{d}=0.53$ ( $w / D=0.28$ ), where $d$ is the minor dimension of the nozzle. When tabs with larger width, w/d $=0.71(\mathrm{w} / \mathrm{D}=0.38)$, are used the jet distortion 
at $\mathrm{x} / \mathrm{D}=1$ and 2 are only slightly more accentuated. This is shown by the pair of pictures in the middle of Fig. 9. However, when the width $\mathrm{w}$ equals the minor dimension, $w / d=1(w / D=$ 0.53 ), a remarkably more pronounced distortion occurs as observed in the lower pair of pictures of Fig. 9.

The pictures for $\mathrm{x} / \mathrm{D}=1$ also show that the curling of the shear layer for the two smaller tab dimensions is commensurate with the vorticity data, i.e., the curling has occurred in the direction of rotation of the streamwise vortices. In other words, the shear layer has curled up as if to indicate the presence of the pair of streamwise vortices with the correct sense of rotation. With $w / d=1$, however, a curling of the shear layer in opposite direction has occurred. It is as if the delta tabs have produced pairs of streamwise vortices having an opposite sense of rotation. This was quite intriguing, conflicted with the vorticity generation mechanism proposed in Ref. 6 , and could only be clarified with the vorticity measurements.

Hot wire data, as in Fig. 8., obtained with the 3:1 rectangular nozzle and two delta tabs having $w / d=1$, are shown in Fig. 10. An inspection of the velocity contours on the left should confirm that the jet distortion here $\left(\mathrm{M}_{\mathrm{j}}=\right.$ 0.31 ) is similar to that observed for $M_{j}=1.63$ in Fig. 9 (note the different orientations of the nozzles in the two figures). The $\omega_{x}$ data, shown on the right of Fig. 10, demonstrate that streamwise vorticity with the expected sense of rotation is indeed produced. However, the streamwise vorticity is distributed over a large extent of the mixing layer. The sheet of streamwise vorticity only curls up on the ends to produce the anomalous impression described in the foregoing. The mechanism determining the direction of curling up remains unclear. However, these observations,

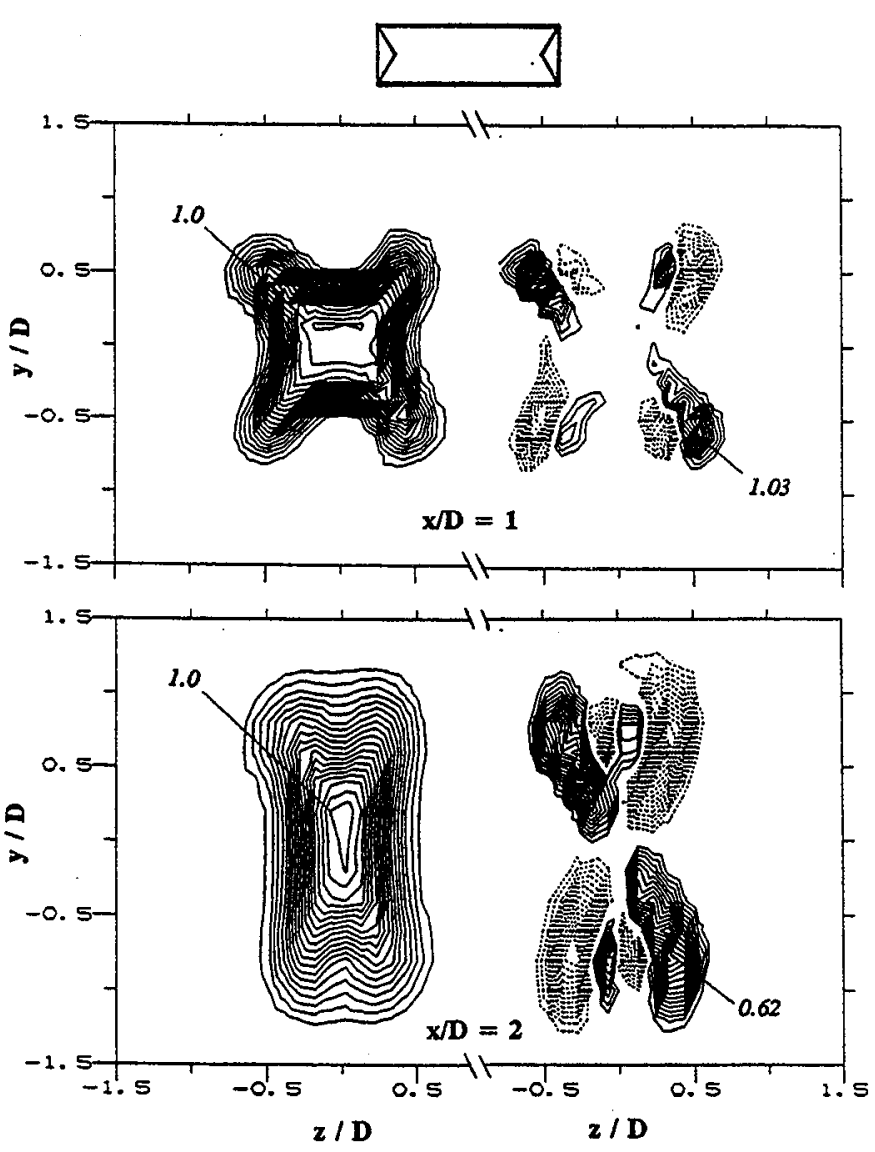

Fig. 10 Contours of $U / U_{j}$ and $\omega_{x} D / U_{j}$, as in Fig. 8, for the 3:1 rectangular jet $(D=6.35 \mathrm{~cm})$ with two large delta tabs $(\mathbf{w} / \mathrm{D}=\mathbf{0 . 5 3})$.

once again, underscore the pitfalls in drawing inferences on vorticity dynamics from flow visualization.

It is clear that the jet in Fig. 10 has gone through an axis switchover by $\mathrm{x} / \mathrm{D}=2$, and elongated enormously in the minor axis plane. This becomes clear by comparison with the data of Fig. 8(b). Comparison of the $\omega_{x}$ data also show that significantly more vorticity has been produced by the larger delta tabs. Recall from the discussion of Fig. 3 that with this tab configuration there also occurs a large increase in the mass fluxes downstream. The corresponding Mach number contours at $x / D=14$, measured with the 


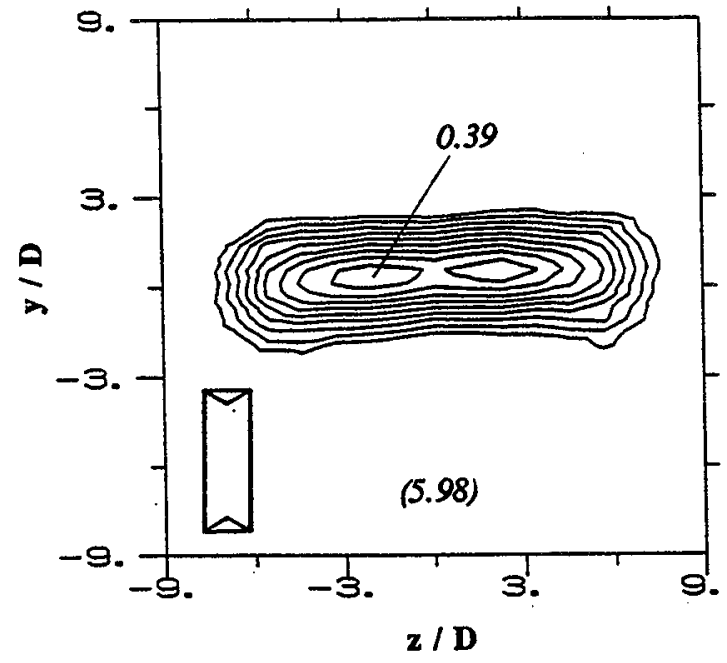

Fig. 11 Mach number contours, as in Fig. 2, for 3:1 rectangular jet $(D=1.47 \mathrm{~cm})$ with two large delta tabs $(w / D=0.53) ; x / D=14, M_{j}=1.63$.

small nozzle at $\mathbf{M}_{\mathbf{j}}=1.63$, are shown in Fig. 11 . Comparison with Figs. 2 and 5 makes it clear that the jet has elongated much more in the minor axis plane, (note the difference in scales.) At $x=$ $14 \mathrm{D}$ the jet half width is about $14 \mathrm{D}$ representing approximately a $50^{\circ}$ flaring of the jet. The maximum Mach number at $\mathrm{x}=14 \mathrm{D}$ is well below that for any other cases in Fig. 2. The mass flux is also quite large compared to the other cases, which has been shown in Fig. 3 .

\section{Concluding remarks}

The overall mixing characteristics of jets from convergent nozzles of different exit shapes have been compared at a jet Mach number of 1.63. Flows from a $3: 1$ elliptic, and $3: 1,4: 1$ and $8: 1$ rectangular nozzles are compared with that from an axisymmetric nozzle. Mixing, as denoted by the mass flux measured at certain downstream locations, is found to be higher for all the asymmetric nozzles compared to the axisymmetric case. Four delta tabs with the axisymmetric nozzle increase the mixing significantly; the fluxes in this case are higher than that produced by any of the asymmetric nozzles. It is emphasized that the normalized mass flux data presented here pertain to underexpanded flows from convergent nozzles at a specific jet Mach number. The flux amplitudes are expected to change with different states of the flow and Mach number. It is possible, however, that the relative trends for the different nozzles and tab configurations would remain the same at the other flow conditions. The latter comment is based on the similarities observed in the data trends between the subsonic and supersonic conditions examined in this study. Also it should be noted that all the jets without tabs had screech which may have resulted in somewhat higher fluxes for those cases, as screech is known to increase mixing. ${ }^{22}$

Use of the tabs with the asymmetric nozzles generally resulted in only small increase in the fluxes, some of the tab configurations even decreased the fluxes. However, two large delta tabs placed on the narrow edges of the 3:1 rectangular nozzle provided fluxes that were the largest among all the cases tried so far. The jet in this case 'fanned out' at a large angle after going through one axis switch.

The axis switching phenomenon occurring with the asymmetric nozzles could be affected by the delta tabs. This was studied in detail with a 3:1 rectangular nozzle. The flow from this nozzle without the tabs produced four dominant streamwise vortices originating from the four corners of the nozzle. These formed two pairs of counterrotating vortices located on the ends of the major axis. Each of the vortex pairs had a sense of rotation so as to eject jet core fluid into the ambient. These vortices faded away by $\mathrm{x} / \mathrm{D}=$ 16; and the jet did not go through an axis switch by that distance. Use of two delta tabs placed on 
the narrow edges of the nozzle overwhelmed the naturally occurring vortices and produced two stronger pairs of vortices having an opposite sense of rotation. The jet in this case went through a rapid axis switch. Two delta tabs placed on the long edges of the nozzle, on the other hand, produced vortices which amalgamated with the naturally occurring vortices. The jet in this case continued to diverge on the major axis plane within the measurement range.

The mechanism proposed in Ref. 12, referred here as $\omega_{\theta}$-induced dynamics, explains the rapid axis switching for periodically forced asymmetric jets. The axis switching observed with the screeching jets is also thought to be due to this mechanism. However, the $\omega_{\ominus}$-induced dynamics do not explain the observations made for the tab cases. The induced motion of the streamwise vortex pairs, referred to as the $\omega_{x^{-}}$ induced dynamics, is believed to hold the key for the latter cases. The sense of rotation of the vortex pairs determines if there would be an axis switch or not. Specifically, two vortex pairs located on the ends of the major axis and having the sense of rotation so as to eject jet core fluid, would resist axis switching. On the other hand, two vortex pairs having an opposite sense of rotation, as produced by the delta tabs placed on the narrow edges of the nozzle, would augment the axis switching process.

It should be recognized that since streamwise vortices occur naturally in asymmetric jets, the $\omega_{\mathrm{x}}$-induced dynamics should be operative even without the tabs. It is reasoned that a combination of the $\omega_{\theta}$-induced and $\omega_{x}$-induced dynamics is responsible for the slow axis switching observed with asymmetric nozzles having upstream contractions or long channels. The rapid axis switch observed with asymmetric orifices, on the other hand, is believed to be due to the dominance of the $\omega_{\theta}$-induced dynamics and an absence of the $\omega_{\mathrm{x}}$-induced dynamics. Also, referring back to the discussion of Refs. 26,27 and 28 in $\$ 3.2$, it should be recognized that the streamwise vorticity distribution occurring at the exit of a nozzle is quite sensitive to the upstream geometry of the nozzle. It may be possible to obtain vortex pairs with varying strength with a sense of rotation the same as that observed here. It may also be possible to obtain vortex pairs having an opposite sense of rotation. In the latter case, the $\omega_{\mathrm{x}}$-induced dynamics would reinforce the $\omega_{\theta^{-}}$ induced dynamics causing a much faster axis switching.

Finally, the present results also showed that the curling of the shear layer observed in flow visualization might not necessarily indicate the correct sense of rotation of the streamwise vorticity embedded in the region.

\section{Acknowledgement}

The author would like thank Mr. M.F. Reeder and Prof. M. Samimy of Ohio State University, and Prof. J.F. Foss of Michigan State University for providing help in various forms throughout the course of this work.

\section{References:}

${ }^{1}$ Ahuja K.K. and Brown W.H., "Shear flow control by mechanical tabs", AlAA Paper 89-0994, 1989.

${ }^{2}$ Samimy M., Zaman K.B.M.Q. and Reeder M.F., "Effect of tabs at the nozzle lip on the flow and noise field of an axisymmetric jet", ALAA J., 31(4), p.609, April 1993.

${ }^{3}$ Rogers, C.B. and Parekh, D.E., "Jet mixing enhancement and noise reduction by streamwise vortices", private communication, D.E. Parekh, 1993.

${ }^{4}$ Zhang, S. and Schneider, S.P., "Jet mixing enhancement using tabs: preliminary results of reacting flow visualization experiments", ASME Winter Annual Meeting, New Orleans, Dec. 1993.

${ }^{5}$ Reeder, M.F. and Samimy, M., "The evolution of an axisymmetric jet with vortex generating tabs", ASME Winter Annual Meeting, New Orleans, Dec. 1993. 
- Zaman K.B.M.Q., Reeder M.F., and Samimy M., "Control of an axisymmetric jet using vortex generators", to appear in Physics of Fluids A, Feb. 1994.

${ }^{7}$ Bradbury L.J.S. and Khadem A.H., "The distortion of a Jet by Tabs", J. Fluid Mech., vol. 70, p. 801, 1975.

${ }^{8}$ Tanna H.K., "An Experimental Study of Jet Noise, Part II: Shock Associated Noise", J. Sound \& Vib. vol. 50, p. 429, 1977.

${ }^{9}$ Kobayashi, H., Oinuma, H., Sawamura, T. and Outa, E., "Effects of tab size on supersonic underexpanded cold and heated jet noise suppression and jet thrust loss", ALAA Aeroacoustics Conf. (93-4348), Long Beach, CA, Oct. 1993.

${ }^{10}$ Krothapalli, A., King, C.J. and Strykowski, P.J., "The role of streamwise vortices on sound generation of a supersonic jet", ALAA Aeroacoustics Conf. (93-4320)., Long Beach, CA, Oct. 1993.

"Ho C.-M. and Gutmark E., "Vortex induction and mass entrainment in a small-aspect-ratio elliptic jet", J. Fluid Mech., 179, p. 383, 1987.

${ }^{12}$ Hussain F. and Husain H.S., "Elliptic jets. Part 1. Characteristics of unexcited and excited jets", J. Fluid Mech., 208, p. 257, 1989.

${ }^{13}$ Schadow K.C., Wilson K.J., Lee M.J. and Gutmark E., "Enhancement of mixing in reacting fuel-rich plumes issued from elliptic nozzles", J. Propulsion, 3, p. 145, 1987.

${ }^{14}$ Seiner, J. M. and Krejsa, E.A., "Supersonic jet noise and high speed civil transport", AlAA Paper 89-2358, 1989.

is Trestacoste, N. and Sforza, P., "Further experimental results for three-dimensional free jets", ALAA J., 5(5), May, 1967.

${ }^{16}$ Krothapalli, A., Baganoff, D. and Karamcheti, K., "On the mixing of rectangular jet", J. Fluid Mech., 107, p.201, 1981.

17 Tsuchiya, Y., Horikoshi, C. and Sato, T., "On the spread of reactangular jets", Experiments in Fluids, 4, p. $197,1986$.

${ }^{18}$ Morrison, G.L., Swan, D.H. and DeOtte, R.E., "Development of the mean velocity distribution in rectangular jets", AlAA Paper 92-0505, 1992.

${ }^{19}$ Grinstein, F.F., "Vorticity dynamics in spatially-developing rectangular jets", ALAA Shear Flow Conf. (933286), Orlando, FL, July 1993.

${ }^{20}$ Quinn, W.R., "Streamwise evolution of a square jet cross section", ALAA J., 30(12), Dec. 1992.

${ }^{21}$ Zaman, K.B.M.Q., "Streamwise vorticity generation and mixing enhancement in free jets by 'delta-tabs'", $A L A A$ Shear Flow Conf. (93-3253), Orlando, FL, July 1993.
2 Rice, E.J. and Raman, G., "Enhanced mixing of a rectangular supersonic jet by natural and induced screech", AIAA Shear Flow Conf. (93-3263), Orlando, FL, July 1993.

${ }^{23}$ Bell J.H. and Mehta R.D., "Measurements of the streamwise vortical structures in a plane mixing layer", $J$. Fluid Mech., 239, p. 213, 1992.

${ }^{24}$ Crow S.C. and Champagne F.H., "Orderly structures in jet turbulence", J. Fluid Mech., 48, p.547, 1971.

${ }^{25}$ Bradshaw, P., "Turbulent secondary flows", Ann. Rev. Fluid Mech., 19, p. 53, 1987.

${ }^{2}$ Davis, D.O. and Gessner, F.B., "Experimental investigation of turbulent flow through a circular-to-rectangular transition duct", ALAA J., 30, p. 367, Feb 1992.

${ }^{27}$ Sotiropoulos, F. and Patel, V.C., "Numerical calculation of turbulent flow through a circular-to-rectangular transition duct using advanced turbulence closures", $A L A A$ Fluid Dynamics Conf. (93-3030), Orlando, FL, July 1993. ${ }^{28}$ Miau, J.J., Leu, T.S., Chou, J.H., Lin, S.A. and Lin, C.K., "Flow distortion in a circular-to-rectangular transition duct", ALAA J., 28, p.1447, Aug 1990.

${ }^{29}$ Eskinazi, S., "Vector mechanics of fluids and magnetofluids", Academic Press, New York, 1967.

${ }^{30}$ Gutmark, E. and Schadow, K.C., "Azimuthal instabilities and mixing characteristics of a small aspect ratio slot jet", ALAA Aerospace Sciences Meeting (87-0486), Reno, NV, Jan. 1987. 

Public reporting burden for this collection of information is estimated to average 1 hour per response, including the time for reviewing instructions, searching existing data sources, gathering and maintaining the data needed, and completing and reviewing the collection of information. Send comments regarding this burden estimate or any other aspect of this Davis Highway, Suite 1204, Arlington, VA 22202-4302, and to the Office of Management and Budget, Paperwork Reduction Project (0704-0188), Washington, DC 20503.

\begin{tabular}{|l|l|l}
\hline 1. AGENCY USE ONLY(Leaveblank) & $\begin{array}{c}\text { 2. REPORTDATE } \\
\text { January } 1994\end{array}$ & $\begin{array}{l}\text { 3. REPOAT TYPEANDDATESCOVERED } \\
\text { Technical Memorandum }\end{array}$
\end{tabular}

\section{TITLEANDSUBTITLE}

5. FUNDING NUMBERS

Effect of 'Delta Tabs' on Mixing and Axis Switching in Jets From Asymmetric Nozzles

6. AUTHOR(S)

WU-537-02-22

K.B.M.Q. Zaman

\section{PERFORMING ORGANIZATION NAME(S)AND ADDRESS(ES)}

National Aeronautics and Space Administration

Lewis Research Center

Cleveland, Ohio 44135-3191
8. PERFORMING ORGANIZATION REPORT NUMBER

E-8308

9. SPONSORINGMONITORING AGENCY NAME(S)ANDADDRESS(ES)

10. SPONSORINGMONITORING AGENCYREPORT NUMBER

NASA TM-106450

AIAA-94-0186

National Aeronautics and Space Administration

Washington, D.C. 20546-0001

\section{SUPPLEMENTARY NOTES}

Prepared for the 32nd Aerospace Sciences Meeting and Exhibit sponsored by the American Institute of Aeronautics and Astronautics Reno, Nevada, January 10-13, 1994. Responsible person, K.B.M.Q. Zaman, (216) 433-5888.

12a. DISTRIBUTION/AVAILABILITYSTATEMENT

12b. DISTRIBUTIONCODE

Unclassified-Unlimited

Subject Category 02

13. ABSTRACT (Maximum 200 words)

The effect of delta tabs on mixing and the phenomenon of axis switching in free air jets from various asymmetric nozzles was studied experimentally. Flow visualization and Pitot probe surveys were carried out with a set of small nozzles $(\mathrm{D}=1.47 \mathrm{~cm})$ at a jet Mach number, $\mathrm{Mj}=1.63$. Hot wire measurements for streamwise vorticity were carried out with larger nozzles $(\mathrm{D}=6.35$ $\mathrm{cm}$ ) at $\mathrm{Mj}=0.31$. Jet mixing with the asymmetric nozzles, as indicated by the mass fluxes downstream, was found to be higher than that produced by a circular nozzle. The circular nozzle with four delta tabs, however, produced fluxes much higher than that produced by a asymmetric nozzles themselves or by most of the tab configurations tried with them. Even higher fluxes could be obtained with only a few cases, e.g., with 3:1 rectangular nozzle with two large delta tabs placed on the narrow edges. In this case the jet 'fanned out' at a large angle after going through one axis switch. The axis switching could be either stopped or augmented with suitable choice of the tab configurations Two mechanisms are identified governing the phenomenon. One, as described in Ref. 12 and referred to here as the $\omega_{\theta}$-induced dynamics, is due to differential induced velocities of different segments of a rolled up azimuthal vortical structure. The other is the $\omega_{x}$-induced dynamics due to the induced velocities of streamwise vortex pairs in the flow. While the former dynamics are responsible for rapid axis switching in periodically forced jets, the effect of the tabs is governed mainly by the latter. It is inferred that both dynamics are active in a natural asymmetric jet issuing from a nozzle having an upstream contraction. The tendency for axis switching caused by the $\omega_{\theta}$-induced dynamics is resisted by the $\omega_{x}$-induced dynamics, leading to a delayed or no switchover in that case. In jets from orifices and in screeching jets, the $\omega_{\theta}$-induced dynamics dominate causing a faster switchover.

14. SUBJECTTERMS

Jets; Nozzles; Vortex generators; Streamwise vortices 20

16. PRICE CODE $\mathrm{AO} 3$

\begin{tabular}{|c|c|c|}
\hline $\begin{array}{c}\text { 17. SECURITYCLASSIFICATION } \\
\text { OF REPORT } \\
\text { Unclassified }\end{array}$ & $\begin{array}{c}\text { 18. SECURITY CLASSIFICATION } \\
\text { OF THIS PAGE } \\
\text { Unclassified }\end{array}$ & $\begin{array}{c}\text { 19. SECURITY CLASSIFCATION } \\
\text { OF ABSTRACT } \\
\text { Unclassified }\end{array}$ \\
\hline
\end{tabular}

